\title{
Competition between Covalent Bonding and Charge Transfer at Complex-Oxide Interfaces
}

\author{
Juan Salafranca, ${ }^{1,2, *}$ Julián Rincón, ${ }^{2,3}$ Javier Tornos, ${ }^{1}$ Carlos León, ${ }^{1}$ Jacobo Santamaria, ${ }^{1}$ Elbio Dagotto, ${ }^{2,4}$ \\ Stephen J. Pennycook, ${ }^{5}$ and Maria Varela ${ }^{2,1}$ \\ ${ }^{1}$ Grupo de Física de Materiales Complejos, Universidad Complutense, 28040 Madrid, Spain \\ ${ }^{2}$ Materials Science and Technology Division, Oak Ridge National Laboratory, Oak Ridge, Tennessee 37831, USA \\ ${ }^{3}$ Center for Nanophase Materials Sciences, Oak Ridge National Laboratory, Oak Ridge, Tennessee 37831, USA \\ ${ }^{4}$ Department of Physics and Astronomy, The University of Tennessee, Knoxville, Tennessee 37996, USA \\ ${ }^{5}$ Department of Materials Science and Engineering, The University of Tennessee, Knoxville, Tennessee 37996, USA
}

(Received 20 October 2013; published 14 May 2014)

\begin{abstract}
Here we study the electronic properties of cuprate-manganite interfaces. By means of atomic resolution electron microscopy and spectroscopy, we produce a subnanometer scale map of the transition metal oxidation state profile across the interface between the high Tc superconductor $\mathrm{YBa}_{2} \mathrm{Cu}_{3} \mathrm{O}_{7-\delta}$ and the colossal magnetoresistance compound $(\mathrm{La}, \mathrm{Ca}) \mathrm{MnO}_{3}$. A net transfer of electrons from manganite to cuprate with a peculiar nonmonotonic charge profile is observed. Model calculations rationalize the profile in terms of the competition between standard charge transfer tendencies (due to band mismatch), strong chemical bonding effects across the interface, and $\mathrm{Cu}$ substitution into the $\mathrm{Mn}$ lattice, with different characteristic length scales.
\end{abstract}

PACS numbers: 73.20.-r, 74.20.-z, 74.78.Fk

A detailed understanding of the charge transfer that occurs across semiconductor interfaces has led to the development of two-dimensional electron gases [1], as well as the integer and fractional quantum Hall effect [2-4]. Interfaces between transition-metal oxides (TMOs) have the potential for even richer physics, due to the presence of several competing interactions with similar characteristic energies. The competition between electrostatic effects-similar to those at work in semiconductor heterostructures-and orbital physics characteristic of TMOs can give rise to exotic electronic reconstructions and novel physical behaviors. In heterostructures of $\mathrm{LaAlO}_{3} / \mathrm{SrTiO}_{3}$, the observation of a metalinsulator transition at the interface of these nonmagnetic (bulk) insulators [5] along with superconductivity [6] and magnetism [7]) sparked considerable interest. However, oxide interfaces also bring along many challenges. Ionic defects such as oxygen vacancies might play an important role in determining the electronic structure [8-13]. Understanding and controlling these material-physics issues - and the effect they have on the properties-is essential to fully explore the new functionalities that these fascinating compounds might bring along [14].

Ferromagnetic-superconducting interfaces of $\mathrm{La}_{2 / 3} \mathrm{Ca}_{1 / 3} \mathrm{MnO}_{3} / \mathrm{YBa}_{2} \mathrm{Cu}_{3} \mathrm{O}_{7-\delta}$ (LCMO/YBCO) have attracted much attention. This system is a paradigmatic example of competition between strongly correlated systems with different ground states. It has been proposed, based on the difference between chemical potentials, that electronic charge would be transferred from the manganite to the cuprate $[15,16]$. This mechanism, however, does not consider the details of the interface. The interfacial electronic structure depends on other details, such as the atomic termination [17] for each material. At the LCMO/YBCO interface both a change in the orbital occupation and a net magnetic moment are induced in the cuprate $[18,19]$. Model calculations [20] were able to explain different experimental results regarding the competition between ferromagnetism and superconductivity [21]. However, the effect of charge transfer was not studied. Very recently, cross-sectional scanning tunneling microscopy measurements have suggested [22] that charge transfer takes place with a characteristic length scale of $\sim 1 \mathrm{~nm}$. However, the interpretation of these measurements is unclear. Further work aimed at studying the electronic structure-including charge distributions - and the importance of interface and bulk effects is necessary to gain full understanding of properties of these interfaces.

In this Letter, we present a combined experimental and theoretical study of the (100) LCMO/YBCO interface. The unique capabilities of scanning transmission electron microscopy (STEM), in combination with electron energy-loss spectroscopy (EELS), allow us to identify the precise chemical terminations, and to establish an oxidation state profile with sub-nanometer resolution. We find an anomalous charge redistribution, with a nonmonotonic behavior of the occupancy of $d$ orbitals in the manganite layers, as a function of distance to the interface. Model calculations indicate that this profile is a result of the competition between standard charge transfer tendencies, strong bonding effects across the interface, and $\mathrm{Cu}$ substitution into the $\mathrm{Mn}$ lattice. We also study the effect of oxygen vacancies, electron-electron interactions, and the polar discontinuity mechanism, and we find that their effect is not important in reproducing the shape of the charge distribution. 
A high magnification $Z$-contrast image of a $\mathrm{La}_{0.7} \mathrm{Ca}_{0.3} \mathrm{MnO}_{3} / \mathrm{YBCO} / \mathrm{La}_{0.7} \mathrm{Ca}_{0.3} \mathrm{MnO}_{3}$ trilayer [23] is shown in Fig. 1(a). Interfaces are sharp and coherent, and the structural quality of the samples is high. Occasionally, interface steps one unit cell high are observed [24]. The structure is, however, unusual near the interfaces. Figure 1(b) shows the $3 \mathrm{~d}$-metal-to-3d-metal distance along the growth direction $(z)$ for the whole image, while the lattice parameter in the manganite is constant all the way to the interface, the YBCO intracell distances exhibit a nonlinear relaxation with a characteristic length of one or two unit cells. The $\mathrm{CuO}_{2}$ planes in the first and second unit cells by the interface move further apart from each other, while the $\mathrm{CuO}_{2}$ plane to $\mathrm{CuO}$ chain distance is somewhat decreased. These nonlinear effects are likely related to the relaxation of epitaxial strain due to lattice mismatch [25]. Also, both top and bottom interfaces lack $\mathrm{CuO}$ chains (this atomic plane exhibits the darkest contrast) [24]. Confirmation of the stacking sequence can be obtained by EEL spectrum images. Figure 1(c) shows atomic resolution maps of the $\mathrm{O} K, \mathrm{Mn} L_{2,3}, \mathrm{Ba} M_{4,5}$ and La $M_{4,5}$ absorption edges, respectively. The overlay of $\mathrm{Mn}$ (red), $\mathrm{La}$ (green), and $\mathrm{Ba}$ (blue) maps proofs that at both interfaces a $\mathrm{Ba}-\mathrm{O}$ plane is facing a $\mathrm{Mn}-\mathrm{O}$ plane. In the predominant termination, no interfacial $\mathrm{CuO}$ chains are observed. Spectroscopic data, including line scans such as the one in Fig. 1(c), show that the interfaces are chemically
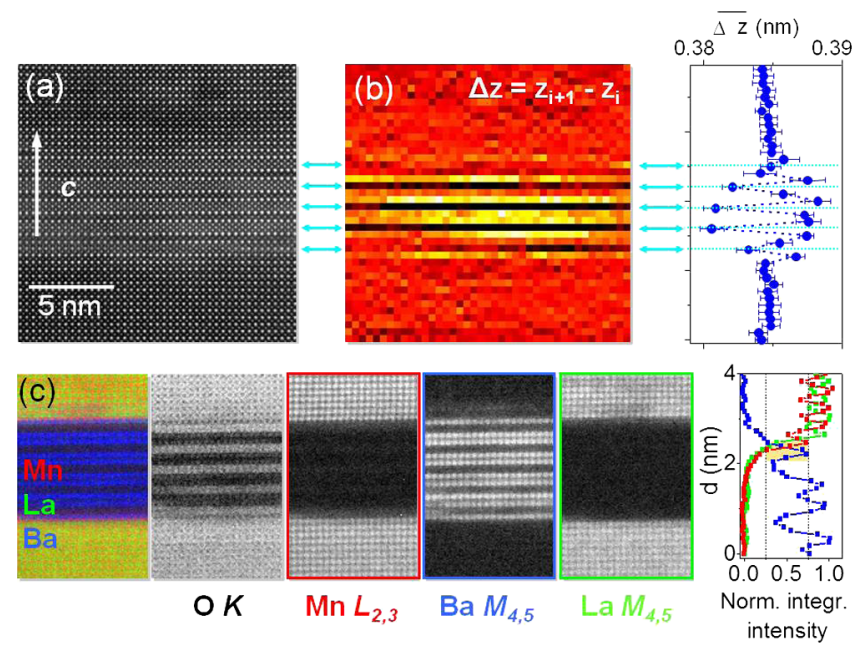

FIG. 1 (color online). (a) High-resolution, Z-contrast image of a LCMO/YBCO/LCMO trilayer. (b) Map of transition metal spacings, $\Delta z$, along the c direction, with a lateral average of the image (right). $\mathrm{CuO}_{2}$ biplanes are characterized by a smaller distance (dark stripes). (c) RGB compound image (left) and EELS maps of the integrated intensity of $\mathrm{O} K$ edge, $\mathrm{Mn} L_{2,3}$, Ba $M_{4,5}$ and $\mathrm{La} M_{4,5}$ edges, as labeled. The RGB imaged is obtained by overlaying the Mn (red), La (green) and Ba (blue) maps. The right panel shows the normalized integrated intensities of the Mn $L_{2,3}$ (red), Ba $M_{4,5}$ (blue), and La $M_{4,5}$ (green) across a LCMO (top)/YBCO(bottom) interface, extracted from an EELS line scan. An orange rectangle marks the width of a perovskite unit cell block at the interface. abrupt within the precision of the technique, limited by the unavoidable formation of amorphous layers during specimen preparation (Supplemental Material [33]). The abrupt interface is consistent with previous x-ray work [23].

These structural changes have a direct impact on the electronic properties, which can also be analyzed from EELS. The EELS fine structure reflects the details of the unoccupied density of states. In particular, the $\mathrm{O} K$ edge fine structure correlates with the electronic doping in both manganites and cuprates $[21,26]$, as does the intensity ratio between the $L_{2}$ and $L_{3}$ edges of Mn. Figure 2(a) shows the variation in the $\mathrm{O} K$ edge across several LCMO (red)/ YBCO (blue) bilayers superimposed over a low magnification image of a YBCO/LCMO superlattice. Figure 2(b) shows the actual background subtracted spectra, acquired while moving from the middle of a LCMO layer into the adjacent YBCO layer. Changes both in the intensity of the main peak $(\approx 535 \mathrm{eV})$, the prepeak $(\approx 530 \mathrm{eV})$ and its position (dashed lines) can be observed. The profiles for the prepeak intensity and the position of the edge onset are shown in Fig. 2(c). These quantities are not the same.

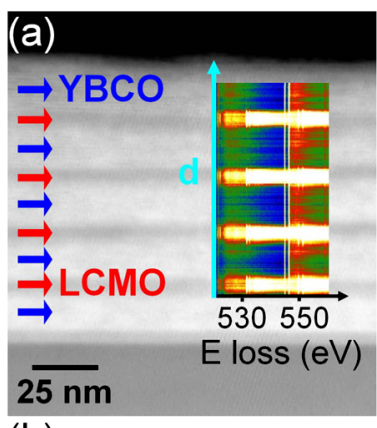

(b)

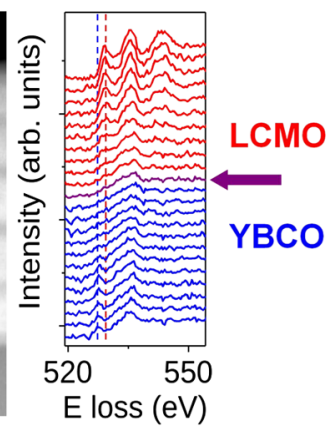

E loss (eV)

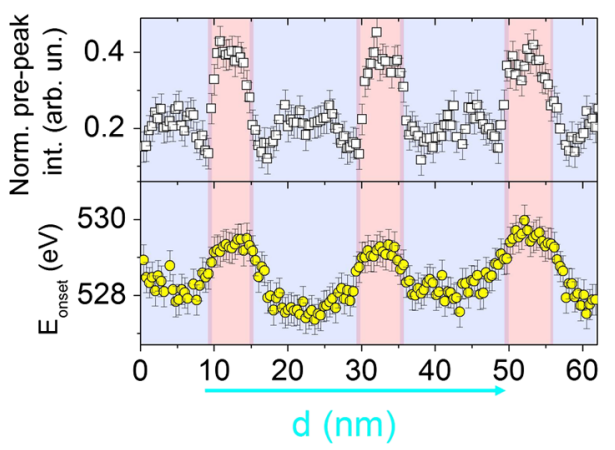

FIG. 2 (color online). (a) Z-contrast image of a LCMO/YBCO multilayer on a (100) $\mathrm{SrTiO}_{3}$ substrate. Arrows mark LCMO (red) and YBCO layers (blue). The inset shows an EELS linescan acquired along the growth direction. The right panel shows the energy range corresponding to the $\mathrm{O} K$ edge across one of the YBCO-LCMO interfaces in the line scan. Dashed lines marked the position of the O prepeak for LCMO and YBCO away from the interface. (b) Prepeak intensity (top), and the edge onset position (bottom) along the growth direction, marked with a light blue arrow. Some data adapted from previous work (see Supplemental Material [33] for details). 
The changing onset of the absorption edge is produced by the shift in the core-level energies. It is reasonable to assume that the bulk chemical potential of YBCO is around $2 \mathrm{eV}$ lower than the LCMO bulk chemical potential [15], and a net transfer of electrons from manganite to cuprate takes place until the chemical potentials reach equilibrium, shifting the core levels a similar energy. On the other hand, the prepeak intensity reflects the occupation of specific orbitals, as we present later; it has been found to be proportional to the oxidation state of the transition metal in manganites [27], and it is also correlated with the hole carrier density in YBCO [26]. Therefore, Fig. 2 reveals both the formal Mn valence within the LCMO layers and the hole doping in the YBCO. Near the interface, the prepeak intensity in YBCO decreases indicating a reduced hole density (i.e., the electron doping increases). The prepeak is also reduced within a $\mathrm{nm}$ in the LCMO side of the interface, sign of a reduced Mn oxidation state [27], also consistent with the sign of the difference in bulk chemical potential.

However, a more refined analysis of the charge profiles in manganite layers of different thicknesses [Fig. 3(a)] reveals surprises. These profiles have been calculated by subtracting the Mn valence measured from the $L_{23}$ intensity ratio [27] from the nominal +3.3 expected according to the chemical doping. A few nanometers away from the interface, LCMO shows a deficit of electrons, as expected to compensate for the extra electrons in YBCO. It is worth noting that these experiments were carried out at room temperature where YBCO is a bad metal, and LCMO is an insulator. Screening in the insulating phase is significantly less efficient, resulting in charge transfer with a much larger characteristic length. The overall profile is compatible with an electron reconstruction driven by the chemical potential mismatch of the two materials [15]. However, the region closest to the interface shows an electron enrichment at both sides of the interface. This unexpected behavior is in principle incompatible with usual semiconductorlike physics, implying the appearance of an additional energy scale competing with charge transfer effects.

In order to explore the origin of the unexpected charge distribution, we turn to model calculations. We concentrate on two basic interactions: the kinetic energy of conduction and
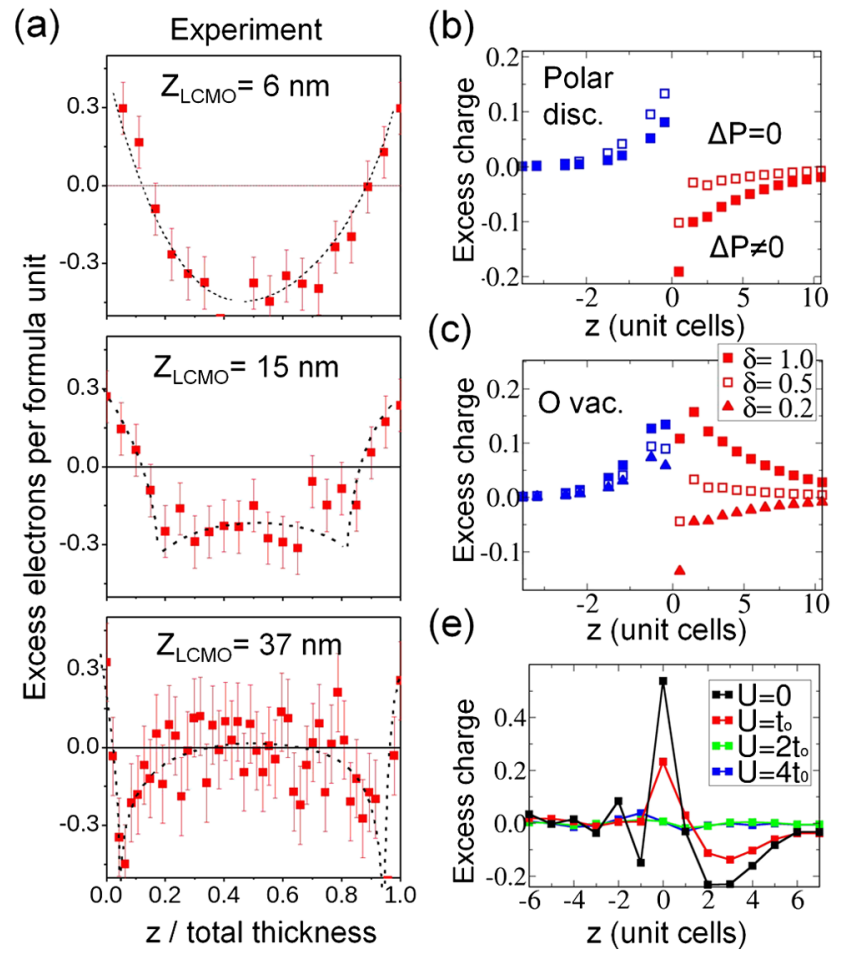

(d) Mn-Cu Hybridazation
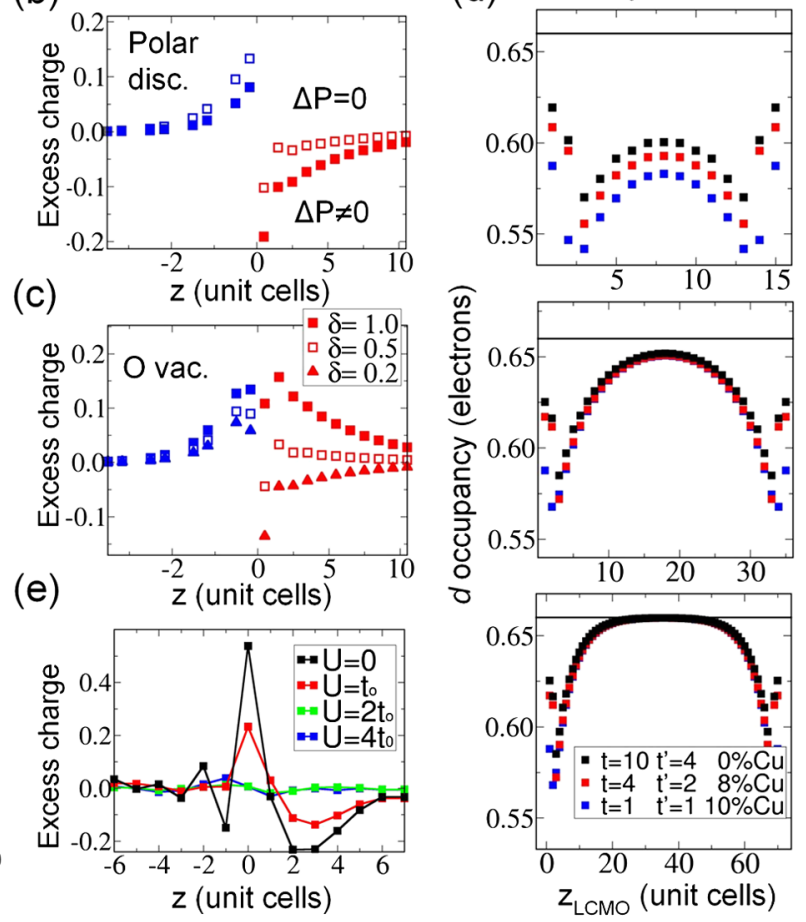

FIG. 3 (color online). Experimental and theoretical charge profiles. (a) Experimental charge profiles across the LCMO layer in multilayers with different thicknesses $Z_{\mathrm{LCMO}}$. Some data adapted from previous work (see Supplemental Material [33] for details). (b) Results of the model calculations, including (empty symbols) and excluding (full) the polar discontinuity at the interface. Blue symbols correspond to YBCO and red symbols correspond to LCMO. Notice that polar discontinuity cannot account for the electron enrichment at LCMO near the interface. (c) Effect of oxygen vacancies. $\delta$ labels the oxygen deficiency in the Mn plane closest to the interface (of chemical formula $\mathrm{MnO}_{2-\delta}$ ). Although oxygen vacancies dope the interface with electrons, the charge profiles in the LCMO layer decrease monotonically to zero, unlike the experiments in (a). (d) Effect of strong hybridization of $\mathrm{Cu}$ and $\mathrm{Mn}$ orbitals in the model and of hybridization together with $\mathrm{Cu}$ substitution in LCMO first atomic plane (as indicated), showing the nonmonotonic charge profile as in (a). (e) Effect of Hubbard $U$ interaction, showing a charge-transfer scenario for $U \leq t_{0}$. Details about the model in the main text and Supplemental Material [33]. 
valence electrons-due to the hybridization of $d$-like orbitals - and the Coulomb interaction among them and with the ions and core electrons. The effective dielectric constant in YBCO (a metal at room temperature) is chosen much larger than in LCMO (an insulator, details in the Supplemental Material [33]). To model the kinetic energy, the two $e_{g}$ orbitals are important in both manganites [28] and in YBCO near the interface [18,30]. Therefore, we have considered a two-orbital tight binding model with effective hopping and electronic interactions $\left(t_{0} \approx 0.5 \mathrm{eV}\right.$, the manganite-bulk hopping parameter is taken as the energy unit [29], see Supplemental Material [33]). The effective values of the hopping parameter in the $z$ direction across the interface, and in the first manganite layer, $t$ and $t^{\prime}$, might be strongly affected by interface effects, such as the observed lattice relaxations [Fig. 1(b)]. They are the most important parameters in this work, because we use them to explore interface effects in the electronic structure and charge distributions.

Let us now consider possible causes for the atypical charge distribution, starting with the polar discontinuity effect that arises at the interface of two materials with different formal polarizations [9]. In this situation, the electric displacement field grows with increasing layer thickness, unless a transfer of charge towards the interface occurs. This effect is implicitly included in our model, where the potential is calculated by assigning to each atomic plane the charge corresponding to the Wannier functions centered in that plane. One way to isolate the effect of polar discontinuity is to get rid of the formal polarization in each unit cell of the different materials. We can do so by substituting all charge in the different unit cells of each material by a point charge with a value that equals the net charge within each unit cell (the exact value determined by the self-consistent calculation). We place these charges in between the $\mathrm{CuO}_{2}$ biplanes, and the $\mathrm{MnO}_{2}$ planes of cuprate and manganite. Then, the Coulomb potential produced does not depend on the particular termination of any material, thus eliminating the effect of polar discontinuity. However, Fig. 3(b) shows that this particular interface termination enriches the LCMO side of the interface with holes (instead of electrons as in the experiments). Therefore, polar discontinuity is insufficient to understand the phenomena discussed here.

Consider now the influence of oxygen vacancies near the interface. The presence of a significant number of oxygen vacancies is unlikely because the samples are grown in a high-oxygen pressure environment [23]. However, oxygen vacancies (difficult to detect) dope the system with electrons. Furthermore, in epitaxial thin films they can help releasing strain. In order to include them in the model, we adjust the formal charge of the first $\mathrm{MnO}_{2}$ plane (see Supplemental Material [33]) to the charge that corresponds to $\mathrm{MnO}$, while preserving charge neutrality. Polar discontinuity effects are properly included. The resulting charge profile is shown in Fig. 3(c), some general features similar to the experiment are found in the YBCO region; however, there is an important difference in the LCMO region, since the experimental profile has a nonmonotonic behavior.

More complex vacancy distributions are possible, but there is a limitation on the effect of vacancy doping. By applying Gauss's law-and assuming translation invariance parallel to the interface-, it is possible to show that whether the energetics favor electrons or holes near the interface further away the electrostatic interactions would make the charge density tend to the bulk value, creating a monotonic profile. This is true regardless of the values of material dependent dielectric constants, which determine the decay lengths of the charge profiles but not the general features. Therefore, electrostatic effects alone are simply unable to reproduce the experimental nonmonotonic profile.

We turn our attention to the effect of covalent bonding across the interface (due to the strong overlap between the orbitals at both sides) [30]. This effect can be included in the model by increasing the hopping across the interface $(t)$. Additionally, we also consider the changes in the hopping between orbitals in the first two layers of the manganite $\left(t^{\prime}\right)$. An increased hopping across the interface is supported by experiments showing orbital reconstruction [18], and a strong magnetic coupling between $\mathrm{Cu}$ and $\mathrm{Mn}$ moments [19,31]. The results in Fig. 3(d), (for $t=10 t_{0} t^{\prime}=4 t_{0}$ ) show a nonmonotonic charge profile in the manganite layer. Taking also into account possible substitution of $\mathrm{Cu}$ into the $\mathrm{Mn}$ lattice improves the agreement between experiments and calculations, although small to moderate substitution alone cannot account for the nonmonotonic profile by itself (nor can other kinds of chemical disorder, details in Supplemental Material [33]).

Electron-electron interactions do not alter this picture. A numerically exact treatment of the electron-electron interaction is possible via the density matrix renormalization group (DMRG) [32], although dimensionality is then constrained to one. Figure 3(e) illustrates the results of DMRG for a one-dimensional version of the model described above with the inclusion of an interaction term (details in Supplemental Material [33]). Both the charge redistribution and Friedel oscillations are strongly suppressed by electron-electron interaction, and for a value of $U=4 t_{0}$, the charge distribution essentially follows the background charge. Thus, the Hubbard $U$ does not play an important role in explaining our experimental results.

The mechanism by which the large hybridization results in an excess of electrons near the interface can be understood in terms of bonding between $\mathrm{Cu}$ and $\mathrm{Mn}$ orbitals. In the limit of $t \gg t_{\mathrm{Mn}}^{z}, t_{\mathrm{Cu}}^{z}$, a bonding and antibonding orbital will form. The bonding orbital will be occupied making the charge at each of the sites equal to $1 / 2$ electron. In our two-orbital model, a large hopping across the interface between two particular orbitals (in this case $3 z^{2}-r^{2}$ for $\mathrm{Cu}$ and $\mathrm{Mn}$ ) results in a tendency of these orbitals to have a filling close to half an electron per 
orbital. This explains why holes appear in the $3 z^{2}-r^{2}$ orbital in YBCO near the interface [15] — normally full in bulk YBCO-while electrons appear in the $3 z^{2}-r^{2}$ orbital in LCMO, which normally has 0.33 electrons for the doping considered here.

The results in Fig. 3(d) agree with the experimental profile. However, the theoretical profile filling near the interface is never larger than the bulk filling. There are different possible causes for this discrepancy. In the model, the two active Mn orbitals have a nonzero density of states at the Fermi energy, providing enough freedom to screen the extra charge that finds its way to the bonding orbital. A more elaborate model that is able to reproduce the insulating character of LCMO should therefore lead to a better agreement. Among different types of chemical disorder, calculations indicate that small $\mathrm{Cu}$ substitution into the Mn lattice improves the agreement with experiments, if hybridization is also considered (Supplemental Material [33]). Theoretical and experimental results are overall similar and the mechanism due to hybridization of $\mathrm{Cu}$ and $\mathrm{Mn}$ orbitals, possibly complemented by a small $\mathrm{Cu} / \mathrm{Mn}$ substitution in LCMO, provides a rationale for the relative electron enrichment of LCMO near the interface.

In summary, the competition between electronic reconstruction (due to band mismatch of YBCO and LCMO) and the strong bonding across the interface appears responsible for the exotic charge profile observed at YBCO/LCMO interfaces. This competition can be traced down to a combination of electrostatic effects-similar to those at work in semiconductor heterostructures - and orbital physics - characteristic of TMOs. The charge profile and interface physics will depend on the energetics of the $e_{\mathrm{g}}$ levels, and therefore it might be tuned by strain, doping, supeconductivity [40], and electron-lattice interactions [14].

The authors thank Luis Brey for helpful discussions and Masashi Watanabe for the principal component analysis plug-in for Digital Micrograph. Research at ORNL (S. J. P., M. V., E. D., and J. R.) was supported by the U.S. Department of Energy (DOE), Basic Energy Sciences (BES), Materials Sciences and Engineering Division, and through the Center for Nanophase Materials Sciences (CNMS), which is sponsored by the Scientific User Facilities Division, DOE-BES. J.Sal. was supported by the ERC starting Investigator Award, Grant No. 239739 STEMOX and Juan de la Cierva program JCI-2011-09428 (MICINN-Spain). Research at UCM (J.T., C.L., J.San.) was supported by the Spanish MICINN/MINECO through Grants No. MAT2011-27470-C02 and Consolider Ingenio 2010 -CSD2009-00013 (Imagine), and by CAM through Grant No. S2009/MAT-1756 (PHAMA). Computations were supported by the National Center for Supercomputing Applications (U.S. Department of Energy, Contract No. DEAC02-05CH11231).
* Corresponding author.

jsalafra@ucm.es

[1] S. Sze, Physics of Semiconductor Devices (Wiley, New York, 1981), 2nd ed.

[2] R. B. Laughlin, Phys. Rev. Lett. 50, 1395 (1983).

[3] D. C. Tsui, H. L. Stormer, and A. C. Gossard, Phys. Rev. Lett. 48, 1559 (1982)

[4] K. Klitzing, G. Dorda, and M. Pepper, Phys. Rev. Lett. 45, 494 (1980).

[5] S. Thiel, G. Hammerl, A. Schmehl, C. W. Schneider, and J. Mannhart, Science 313, 1942 (2006).

[6] N. Reyren, S. Thiel, A. D. Caviglia, L. F. Kourkoutis, G. Hammerl, C. Richter, C. W. Schneider, T. Kopp, A.-S. Rüetschi, D. Jaccard et al., Science 317, 1196 (2007).

[7] A. Brinkman, M. Huijben, M. van Zalk, J. Huijben, U. Zeitler, J. C. Maan, W. G. van der Wiel, G. Rijnders, D. H. A. Blank, and H. Hilgenkamp, Nat. Mater. 6, 493 (2007).

[8] C. Cantoni, J. Gazquez, F. Miletto Granozio, M. P. Oxley, M. Varela, A. R. Lupini, S. J. Pennycook, C. Aruta, U. S. di Uccio, P. Perna, and D. Maccariello, Adv. Mater. 24, 3952 (2012).

[9] M. Stengel, Phys. Rev. Lett. 106, 136803 (2011).

[10] Z. Zhong, P. X. Xu, and P. J. Kelly, Phys. Rev. B 82, 165127 (2010).

[11] G. Herranz, M. Basletić, M. Bibes, C. Carrétéro, E. Tafra, E. Jacquet, K. Bouzehouane, C. Deranlot, A. Hamzić, J.-M. Broto et al., Phys. Rev. Lett. 98, 216803 (2007).

[12] C. Mitra, C. Lin, J. Robertson, and A. A. Demkov, Phys. Rev. B 86, 155105 (2012).

[13] C. Lin, C. Mitra, and A. A. Demkov, Phys. Rev. B 86, 161102(R) (2012).

[14] E. Dagotto, Science 309, 257 (2005).

[15] S. Yunoki, A. Moreo, E. Dagotto, S. Okamoto, S. S. Kancharla, and A. Fujimori, Phys. Rev. B 76, 064532 (2007).

[16] M. Varela, A. R. Lupini, V. Pena, Z. Sefrioui, I. Arslan, N. D. Browning, J. Santamaria, and S. J. Pennycook, arXiv: cond-mat/0508564.

[17] C. Visani, J. Tornos, N. M. Nemes, M. Rocci, C. Leon, J. Santamaria, S. G. E. te Velthuis, Y. Liu, A. Hoffmann, and J. W. Freeland, Phys. Rev. B 84, 060405 (2011).

[18] J. Chakhalian, J. W. Freeland, H.-U. Habermeier, G. Cristiani, G. Khaliullin, M. Van Veenendaal, and B. Keimer, Science 318, 1114 (2007).

[19] J. Chakhalian, J. W. Freeland, G. Srajer, J. Strempfer, G. Khaliullin, J. C. Cezar, T. Charlton, R. Dalgliesh, C. Bernhard, G. Cristiani et al., Nat. Phys. 2, 244 (2006).

[20] J. Salafranca and S. Okamoto, Phys. Rev. Lett. 105, 256804 (2010).

[21] N. M. Nemes, N. Garcia-Hernandez, S. G. E. te Velthuis, A. Hoffmann, C. Visani, J. Garcia-Barriocanal, V. Pena, D. Arias, Z. Sefrioui, C. Leon, and J. Santamaria, Phys. Rev. B 78, 094515 (2008).

[22] T. Y. Chien, L. F. Kourkoutis, J. Chakhalian, B. Gray, M. Kareev, N. P. Guisinger, D. A. Muller, and J. W. Freeland, Nat. Commun. 4, 2336 (2013).

[23] Z. Sefrioui, M. Varela, D. Arias, V. Peña, C. León, J. Santamaria, J. E. Villegas, J. L. Martinez, W. Saldarriaga, and P. Prieto. Appl. Phys. Lett. 81, 4568 (2002). 
[24] M. Varela, A. R. Lupini, S. J. Pennycook, Z. Sefrioui, and J. Santamaria. Solid State Electron. 47, 2245 (2003).

[25] M. Varela, Z. Sefrioui, D. Arias, M. A. Navacerrada, M. Lucía, M. A. López de la Torre, C. León, G. D. Loos, F. Sánchez-Quesada, and J. Santamaría, Phys. Rev. Lett. 83 3936 (1999).

[26] N. D. Browning, M. F. Chisholm, S. J. Pennycook, D. P. Norton, and D. H. Lowndes, Physica (Amsterdam) 212C, 185 (1993).

[27] M. Varela, M. P. Oxley, W. Luo, J. Tao, M. Watanabe, A. R. Lupini, S. Pantelides, and S. J. Pennycook, Phys. Rev. B 79, 085117 (2009).

[28] E. Dagotto, T. Hotta, and A. Moreo, Phys. Rep. 344, 1 (2001).

[29] J. Salafranca, G. Alvarez, and E. Dagotto, Phys. Rev. B 80, 155133 (2009).

[30] S. Okamoto, Phys. Rev. B 82, 024427 (2010).

[31] Y. Liu, C. Visani, N. M. Nemes, M. R. Fitzsimmons, L. Y. Zhu, J. Tornos, M. Garcia-Hernandez, M. Zhernenkov, A. Hoffmann, C. Leon, J. Santamaria, and S. G. E. te Velthuis, Phys. Rev. Lett. 108, 207205 (2012).
[32] S. R. White, Phys. Rev. Lett. 69, 2863 (1992).

[33] See Supplemental Material at http://link.aps.org/supplemental/ 10.1103/PhysRevLett.112.196802, which includes Refs. [34-39].

[34] M. Varela, C. Leon, J. Santamaria, and S. J. Pennycook, in Multifunctional Oxide Heterostructures, edited by E. Y. Tsymbal, E. R. A. Dagotto, C.-B. Eom, and R. Ramesh (Oxford University Press, Oxford, 2012).

[35] A. Hoffmann, S. G.E. te Velthuis, Z. Sefrioui, J. Santamaria, M. R. Fitzsimmons, S. Park, and M. Varela, Phys. Rev. B 72, 140407R (2005).

[36] J. C. Slater and G. F. Koster, Phys. Rev. 94, 1498 (1954).

[37] E. Dagotto, T. Hotta, and A. Moreo, Phys. Rep. 344, 1 (2001).

[38] J. Salafranca, G. Alvarez, and E. Dagotto, Phys. Rev. B 80, 155133 (2009).

[39] P. W. Peacock and J. Robertson, J. Appl. Phys. 92, 4712 (2002).

[40] B. K. Nikolic, J. K. Freericks, and P. Miller, Phys. Rev. B 65, 064529 (2002). 